\title{
Adaptive Control and Robust MPC for Linearising Longitudinal Vehicle Dynamics for Platooning Applications
}

\author{
Umberto Montanaro $^{1}$, Shilp Dixit ${ }^{1}$, and Saber Fallah ${ }^{1}$ \\ University of Surrey, Guildford GU2 7XH, UK, \\ corresponding author: u.montanaro@surrey.ac.uk
}

\begin{abstract}
Vehicle platooning is a promising cooperative driving vision where a group of consecutive connected autonomous vehicles (CAVs) travel at the same speed with the aim of improving fuel efficiency, road safety, and road usage. To achieve the benefits promised through platooning, platoon control algorithms must coordinate the dynamics of CAVs such that the closed-loop system is stable, errors between consecutive vehicles do not amplify along the string, and the time for re-establish the platoon formation to changes in the operating conditions does not diverge when the number of CAVs increases. Linear longitudinal vehicle dynamics are often assumed in the literature to guarantee such stringent platoon control requirements and they can be attained by equipping vehicles in the fleet with mid-level control systems. However, model uncertainties and disturbances can jeopardise the tracking of the reference linear behaviour. Hence, this paper presents for the first time, at the best of the authors' knowledge, the design and the performance of an adaptive control strategy and a robust model predictive control method as possible solutions for the mid-level control problem. Numerical results confirm that both control techniques are effective at imposing the dynamics of a linear time-invariant system to the longitudinal vehicle motion and they outperform model-based feedback linearisation methods when the parameters of the nonlinear longitudinal vehicle model are affected by uncertainties.
\end{abstract}

Keywords: Longitudinal vehicle control, adaptive control, model predictive control

\section{Introduction}

A vehicle platoon is a group of two or more consecutive Connected Automated Vehicles $(\mathrm{CAVs})$, also denoted as a string of vehicles, travelling along a highway in the same lane with a short inter-vehicle distance and at the same velocity. By organising CAVs in platoons it is possible to improve road transportation by (i) increasing traffic flow while reducing traffic shock waves, (ii) reducing fuel consumption and pollutant emissions, (iii) improving road safety, and (iv) enhance drivers comfort [1]. 
To successfully achieve the benefits promised by vehicle platooning, platoon control algorithms, which exploit vehicle state of the CAVs in the fleet shared via communication channels, are used with the aim of imposing internal and string stability [1]. Individual stability is required for imposing the platoon behaviour (i.e., zero inter-vehicular distance error between consecutive vehicles while imposing the leader's speed to each vehicle). The convergence speed to the synchronous vehicle motion, also known as stability margin, is used to evaluate the time for the platoon to recover the cooperative motion with respect to variations in the operating conditions and it is desired to keep it bounded despite the number of the vehicles in the fleet. Finally, string stability refers to ability of the platoon to not amplify the effect of the disturbances when propagating downstream along the vehicle string. To guarantee platoon stabilities and stability margin, platoon control algorithms are usually designed by assuming that the longitudinal vehicle dynamics can be modelled as a Liner Time Invariant (LTI) (see [2] and references therein). However, nonlinear drag forces, rolling resistance and road slope acting on the vehicle make the longitudinal vehicle model nonlinear. These vehicle nonliearities together with parameter uncertainties can be considered as disturbances acting on the LTI vehicle model, thus a possible approach to achieve platooning while rejecting such unmodelled dynamics is through the design of robust platoon control algorithms (see for instance [3], [4], [5]). Alternatively, control systems, referred hereafter to as mid-level controllers, can be used to linearise the vehicle model to fulfil the linearity assumption. In this framework, model-based controllers can be used for cancelling vehicle nonlinearities. However, parameters uncertainty, unmodelled dynamics and disturbances can limit the effectiveness of model-based solutions to linearise the vehicle model, and thus the achievement of the platoon requirements. Consequently, robust control methods must be exploited to impose the desired linear vehicle behaviour. In this paper, the problem of imposing the linear behaviour to the longitudinal vehicle dynamics is recast a tracking problem of the states of an LTI system (see also the control architecture in Figure 1 where $a_{\text {des }}$ is the desired vehicle acceleration provided by platoon controllers, denoted in the figure as Upper Level Controller).

Two control algorithms are designed to robustly steer the vehicle state towards the reference linear dynamics despite of vehicle parameter uncertainties, i.e., the adaptive strategy in [6] for single mode systems, and the robust model predictive control (RMPC) strategy in [7]. It is remarked that, for the adaptive algorithm, the control gains vary based on the actual vehicle response, thus it does not require any knowledge of the plant parameters and disturbances. However, the adaptive strategy does not allow to systematically constrain the control action in a given desired set, thus limitations of actuation systems cannot be considered explicitly. On contrary, the RMPC strategy optimises the control action such that constraints on the system state and control action are always satisfied. It is noted that, also the RMPC can reject disturbances and parameter uncertainties, but its design requires a precise knowledge of the bounds of the vehicle parameters. Furthermore, the design of the RMPC might not be possi- 


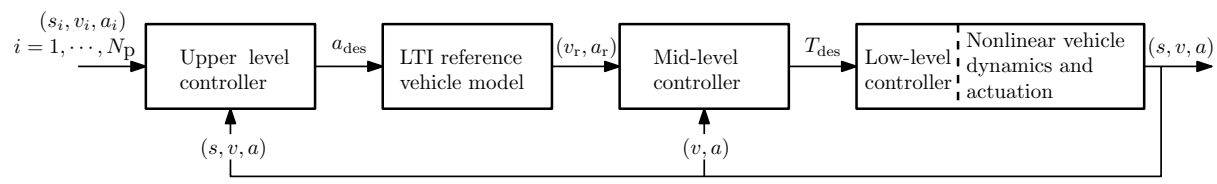

Fig. 1: Control scheme. $v_{r}$ and $a_{r}$ are the reference velocity and acceleration; $s, v$ and $a$ are the actual vehicle position, velocity and acceleration, respectively; $a_{\text {des }}$ is the input to the reference LTI model which is provided by the platoon control (upper level controller) based on the current state of the vehicle and the states of the other platoon members $i=1 \ldots N_{p}$, with $N_{p}$ being the total number of platoon vehicles; $T_{\text {des }}$ is the driving/braking torque (control action) actuated by the low-level controller.

ble in the case of large parameter uncertainties which might make the control set of the underlying optimization problem empty. Consequently, the selected control algorithms are complementary in terms of advantages and disadvantages and they are compared for the first time, at the best of the authors' knowledge, for imposing a linear behaviour to the nonlinear vehicle dynamics. The effectiveness of the control methods to impose the linear behaviour is investigated numerically and advantages and disadvantages of each strategy are pointed out. Moreover it is also shown that in presence of parameter uncertainties, full-state feedback linearisation method may fail, thus confirming the need of more robust linearisation techniques.

The rest of the paper is organised as follows. The vehicle model and the control objective are formulated in Section 2, the the adaptive and RMPC algorithms are presented in Section 3 and Section 4, respectively, the numerical validation is carried out in Section 5 while conclusions are drawn in Section 6 .

\section{Vehicle model and control objective}

For the design of the adaptive and robust control methods the nonlinear longitudinal vehicle model presented in [8] have been considered. After some algebraic manipulation the model in [8] can be recast as the following nonlinear system

$$
\left\{\begin{array}{l}
\dot{v}=a \\
\dot{a}=-\frac{1}{\tau} a-\frac{C_{A}}{\tau M} v^{2}-2 \frac{C_{A}}{M} v a-\frac{g}{\tau} f+\frac{\eta_{T}}{\tau M R_{W}} T_{\mathrm{des}}
\end{array}\right.
$$

where $v$ and $a$ are the velocity and the acceleration of the vehicle, respectively, $M$ is the vehicle mass, $C_{A}$ is the coefficient of the aerodynamic drag, $g$ is the gravity constant, $f$ is the coefficient of the rolling resistance, $u=T_{\text {des }}$ is the control action and represents the desired driving/braking torque, $\tau$ is the inertial lag of the vehicle longitudinal dynamics, $R_{W}$ is the tire radius and $\eta_{T}$ is the mechanical efficiency of driveline. Note that in the derivation of system (1), the system parameters and the road-slope have been considered constant. Moreover, each parameter is supposed unknown but belonging to a known bounded set 
(i.e., for each parameter $p_{j}$ of model $(1), p_{j} \in\left[p_{j}^{\min }, p_{j}^{\max }\right]$ with $p_{j}^{\min }$ and $p_{j}^{\max }$ being known constants).

The control objective is to impose to the system (1) with uncertain parameters the dynamics of a linear reference model of the form

$$
\dot{v}_{r}=a, \quad \dot{a}_{r}=-\frac{1}{\tau_{0}} a+\frac{1}{\tau_{0}} a_{\mathrm{des}}
$$

where $v_{r}$ and $a_{r}$ are the linear velocity and acceleration to impose, $a_{\mathrm{des}}$ is the desired/reference vehicle acceleration provided by the upper-level platoon controller, and $\tau_{0}$ is the nominal intertial lag of the vehicle powertrain system.

\section{Adaptive control}

The adaptive solution used to impose the linear behaviour (2) to system (1) is that presented in [6] under the assumption that only one mode of the system exists. It is noted that the adaptive solution does not require also the knowledge of the upper and lower bounds of the system parameters (i.e., $p_{j}^{\min }$ and $p_{j}^{\max }$ ). The control action is

$$
\begin{gathered}
T_{\mathrm{des}}=\phi^{T}(t) \widehat{\theta}(t), \\
\varphi^{T}=\left[-\Phi^{T} \varphi_{a}-\lambda S\right], \Phi^{T}=-\left[a v^{2} v a 1\right], \varphi_{a}=-\dot{a}_{r}+\lambda e_{a}, \mathcal{S}=e_{a}+\lambda e_{v}
\end{gathered}
$$

where the $e_{v}=v-v_{r}, e_{a}=a-a_{r}, \lambda$ is a positive control parameter and $\widehat{\theta}$ are the adaptive gains which adapt to the vehicle response in accordance with the following adaptive mechanism

$$
\dot{\hat{\theta}}=-\Gamma \mathcal{S} \varphi
$$

with $\Gamma \in \mathbb{R}^{5 \times 5}$ being a positive weight matrix. The adaptive weights have been chosen as $\Gamma=\operatorname{diag}(40,0.02,10,0.5)$ and $\lambda=10$.

\section{Robust Model Predictive Control}

For the application of the robust MPC, system (1) is expressed first as an affine parameter varying system with respect to the vehicle speed with unknown parameters and then discretised by using the forward Euler method with sampling time $T_{s}=0.1 \mathrm{~s}$. Hence, the vehicle dynamics are expressed as

$$
x(k+1)=A_{d}(v) x(k)+B_{d} u(k)+F_{d},
$$

where $x=\left[x_{1}, x_{2}\right]^{T}$ with $x_{1}=v$ and $x_{2}=a, v \in\left[v_{\min }, v_{\max }\right]$ with $v_{\min }$ and $v_{\max }$ are known upper and lower bounds of the vehicle speed, $a \in\left[a_{\min }, a_{\max }\right]$ with $a_{\min }$ and $a_{\max }$ are known upper and lower bounds of the vehicle acceleration, 
$A_{d}(v)=\mathcal{I}_{2}+T_{s} A_{c}(v)$, with $\mathcal{I}_{2}$ being the unit matrix in $\mathbb{R}^{2 \times 2}, B_{d}=T_{s} B_{c}$ and $F_{d}=T_{s} F_{c}$ with

$$
A_{c}=\left[\begin{array}{c}
0 \\
-\frac{C_{A} v}{\tau M}-\left(\frac{1}{\tau}+\frac{2 C_{A} v}{M}\right)
\end{array}\right], B_{c}=\left[\begin{array}{c}
0 \\
\frac{\eta_{T}}{\tau M R_{W}}
\end{array}\right], F_{c}=\left[\begin{array}{c}
0 \\
-\frac{g}{\tau}
\end{array}\right] .
$$

As both, the variation of the parameters assumed in Section 2 and velocity in (6) are bounded, the entries of the dynamic and input matrix are bounded and can be averaged as in [9] (chapter 3). Hence, system (6) can be recast as an LTI system subjected to a disturbance as

$$
x(k+1)=\bar{A} x(k)+\bar{B} u+w,
$$

where $\bar{A}$ and $\bar{B}$ are the constant average matrices and $w=\left(A_{d}(v)-\bar{A}\right) x+$ $\left(B_{d}(v)-\bar{B}\right) u+F_{d}$. As all the entries of $A_{d}(v), B_{d}(v)$ and $F_{d}$ are bounded, and the system input $u \in\left[u_{\min }, u_{\max }\right]$ with $u_{\min }$ and $u_{\max }$ as the upper and lower bounds of control action, the resultant $w$ is also bounded [9]. The states, inputs, and disturbance signals can be represented using a set notation given by $x \in \mathcal{X} \subseteq \mathbb{R}^{2}, u \in \mathcal{U} \subseteq \mathbb{R}^{1}$, and $w \in \mathcal{W} \subseteq \mathbb{R}^{2}$. Moreover, for nominal system dynamics described as

$$
\bar{x}(k+1)=\bar{A} \bar{x}(k)+\bar{B} \bar{u}
$$

the error between system (8) and (9) defined as $e \triangleq(x-\bar{x})$, a stabilising controller $K \in \mathbb{R}^{2 \times 1}$ can be designed so that $A_{\mathrm{K}}=\bar{A}+\bar{B} K$ is Hurwitz, resulting in stable error dynamics expressed as

$$
e(k+1)=A_{\mathrm{K}} e(k)+w .
$$

The error $e \in \mathcal{Z}$ where $\mathcal{Z} \subseteq \mathbb{R}^{2}$ is a robust positively invariant set [10], such that

$$
A_{\mathrm{K}} \mathcal{Z} \oplus \mathcal{W} \subseteq \mathcal{Z}
$$

which results in tightened state and input constraints for the nominal dynamics in (9) given as $\bar{x} \in \overline{\mathcal{X}}=\mathcal{X} \ominus \mathcal{Z}, \bar{u} \in \overline{\mathcal{U}}=\mathcal{U} \ominus K \mathcal{Z}$. Thus, the robust MPC framework described in [7] can be applied to control the system represented by (1). The resultant constrained optimisation problem is given as

$$
\begin{aligned}
\min _{\bar{u}(k), \theta, \bar{x}} & V_{N}(\bar{x}, \bar{u}(k), \theta ; x, \hat{x}) \\
& \text { subject to } \\
& \bar{x} \in x \oplus(-\mathcal{Z}) \\
& \bar{x}(k) \in \overline{\mathcal{X}} \\
& \bar{u}(i) \in \overline{\mathcal{U}} \\
& \bar{x}_{k+1}=\bar{A} \bar{x}(k)+\bar{B} \bar{u}(k), k=0,1, \cdots, N \\
& \left(\bar{x}_{\mathrm{ss}}, \bar{u}_{\mathrm{ss}}\right)=M_{\theta} \theta \\
& (\bar{x}(N), \theta) \in \mathcal{X}_{t}
\end{aligned}
$$

where $N$ is the prediction horizon of the MPC, $x=x(0)$ is the current state of the system, $\hat{x}$ are the target states, $\theta$ is a parameter vector that characterises 
the subspace of steady-states and inputs, and the terminal set $\mathcal{X}_{t}$ is designed as in [7]. The performance index $V_{N}\left(\bar{x}, \bar{u}_{k}, \theta ; x(0), \hat{x}\right)$ is defined as

$$
\begin{aligned}
V_{N}(\bar{x}, \bar{u}(k), \theta ; x, \hat{x})= & \sum_{k=0}^{N}\left[\left\|\bar{x}(k)-\bar{x}_{\mathrm{ss}}\right\|_{Q}^{2}+\left\|\bar{u}(k)-\bar{u}_{\mathrm{ss}}\right\|_{R}^{2}\right]+ \\
& \left\|\bar{x}(N)-\bar{x}_{\mathrm{ss}}\right\|_{P}^{2}+\left\|\bar{x}_{\mathrm{ss}}-\hat{x}\right\|_{T}^{2}
\end{aligned}
$$

The solution of the optimisation problem (12) results in an optimal initial state $\bar{x}^{*}(x, \hat{x})$, an optimal input sequence $\bar{u}^{*}(k)$, and a parametrised steady-state $\theta^{*}(x, \hat{x})$. The net control action applied on the plant is given below.

$$
T_{\mathrm{des}}=\bar{u}^{*}(0, x, \hat{x})+K\left(x-\bar{x}^{*}(x, \hat{x})\right)
$$

The prediction horizon have been chosen as $N=4$, weighting matrices $Q=$ $\operatorname{diag}\left(10^{3}, 10^{2}\right), R=10^{-8}, P$ is the solution to the Lyapunov equation in [7] and can be calculated using commonly available function in MATLAB (e.g., $\operatorname{lqr}(\bar{A}, \bar{B}, Q, R), T=10^{3} \cdot P$, and the nominal control action $K=[660.03,262.3416]$ is utilised.

\section{Main Numerical Results}

For the numerical validation, it was supposed that vehicle parameters deviate from the nominal ones within a given percentage range, e.g., a deviation of $20 \%$ in power-train, $11 \%$ in mass (e.g., due to variations in number of occupants in the car), and $7 \%$ in the powertrain efficiency.

Figure 2 depicts the reference velocity and acceleration provided by a thirdorder LTI vehicle model with time lag of $\tau=0.4 \mathrm{~s}$ and the tracking performance when the full-state feedback linearisation method in [8] is used to achieve the reference linear response in presence of such parameter uncertainties.

The parameter variation mentioned above is used in (6) and (7) to generate the LTI system in (8) and the sets $\mathcal{X}, \mathcal{U}$, and $\mathcal{W}$. Moreover, (10) provides the insight that the set $\mathcal{Z}$ describing the error dynamics depends on $(i)$ the set $\mathcal{W}$ and (ii) matrix $A_{\mathrm{K}}$ [11]. Since the set $\mathcal{W}$ is fixed, the design of the set $\mathcal{Z}$ is via the design of a Hurwitz matrix $A_{\mathrm{K}}$ by choosing an appropriate controller $K$ to ensure stable error dynamics. Figure 3 provides a visual representation for this behaviour where the plot on the left depicts the disturbance set $\mathcal{W}$, the robust positively invariant set $\mathcal{Z}$, state constraints $\mathcal{X}$, and the tightened state constraints $\overline{\mathcal{X}}$ for a given controller while the plot on the right depicts the net input constraints $\mathcal{U}$ and the tightened input constraints $\overline{\mathcal{U}}$. On one hand increasing the dominant eigenvalue beyond $\lambda_{D}=0.90$ results in a large $\mathcal{Z}$ that renders $\overline{\mathcal{X}}=\emptyset$ whereas on the other decreasing the dominant eigenvalue below $\lambda_{D}=0.75$ renders the tightened input constraints $\overline{\mathcal{U}}=\emptyset$ which means that in either case the MPC problem in (12) becomes ill-posed (not feasible). Therefore, for this application the error set obtained using $\lambda_{D}=0.75$ provides a reasonable

compromise between the size of the sets $\overline{\mathcal{X}}$ and $\overline{\mathcal{U}}$ and it is used to solve the MPC problem in (12). 


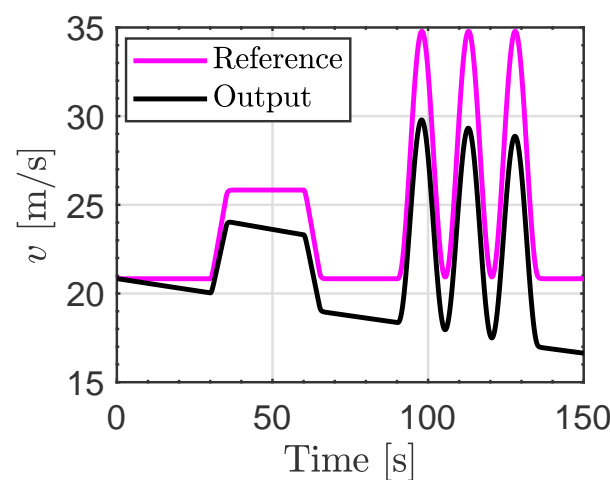

(a)

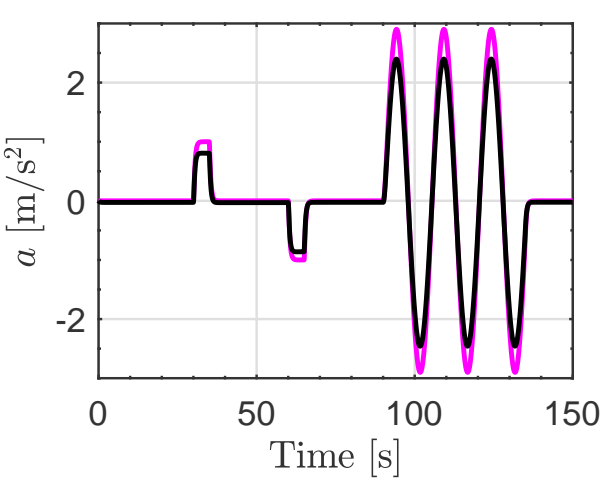

(b)

Fig. 2: Model based results. Reference model (magenta) and vehicle output (black): a) velocity and b) acceleration.

Figure 4 shows the tracking errors and the control action when the adaptive solution and the RMPC strategy are used for imposing the same reference trajectory in Figure 2. It is noted that, both control methods provide small velocity and acceleration tracking errors compared to the amplitudes of the corresponding reference signals, thus confirming their ability to impose linear dynamics to the nonlinear longitudinal vehicle model also in the case of parameter uncertainties. The adaptive solution provides smaller error compared the RMPC mainly because the uncertainty on the system parameters are adaptively compensated as the control gains adapt online based on the actual longitudinal vehicle response. Moreover, over the manoeuvre the adaptive gains remain bounded as shown in Figure 5. However, the adaptive solution does not allow to impose constraints on the rate of variation of the control action (i.e., the driving/ breaking torque that low level controllers must impose), consequently the resulting control action is more aggressive compared to of RMPC especially during rapid variations of the reference vehicle velocity as confirmed in Figure 4c. The trajectories of the systems in a combined state and input space is plotted in the form of a phase portrait in Figure 4d. The plot demonstrates that both the state and input constraints are met for the entire duration of the simulation. Moreover, apart from the large starting transient, the trajectory of the RMPC controller is smoother on the entire state and input space with no spikes or high-frequency oscillations.

\section{Conclusions}

This paper has analysed the performance of two control solutions for imposing the dynamics of an LTI system to the nonlinear vehicle longitudinal dynamics despite system uncertainties for platoon applications. As future work, these 

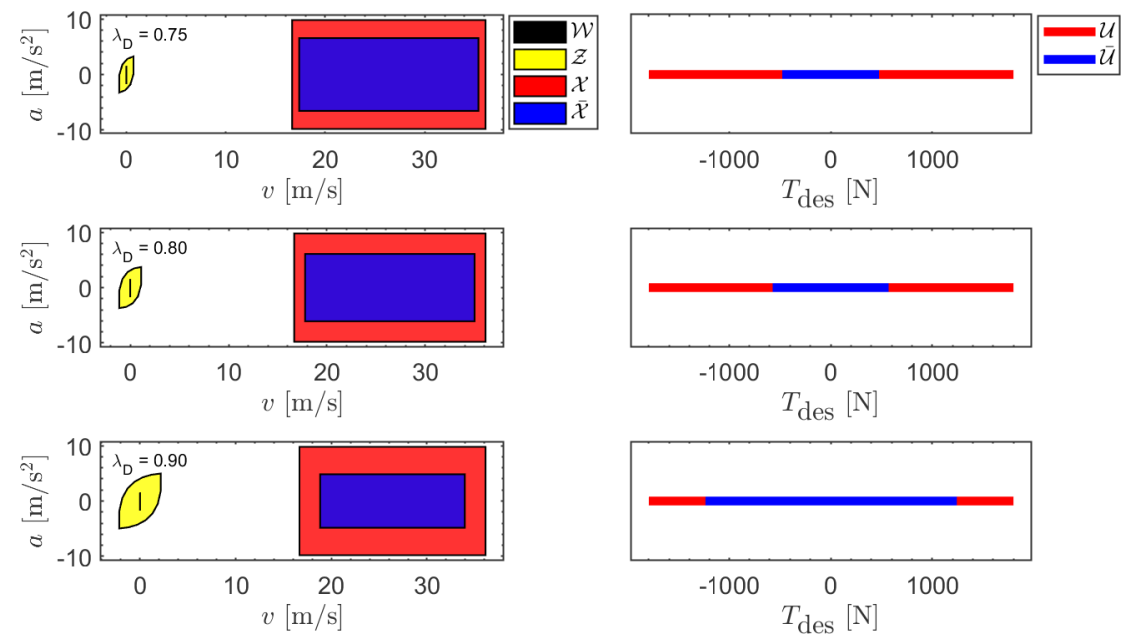

Fig. 3: Error polyhedron and resulting tightened state and input set obtained by changing magnitude of eigenvalue

controllers will be used with platoon control methods based on linear vehicle dynamics which can guarantee internal stability, string stability and stability margin of platoons. 


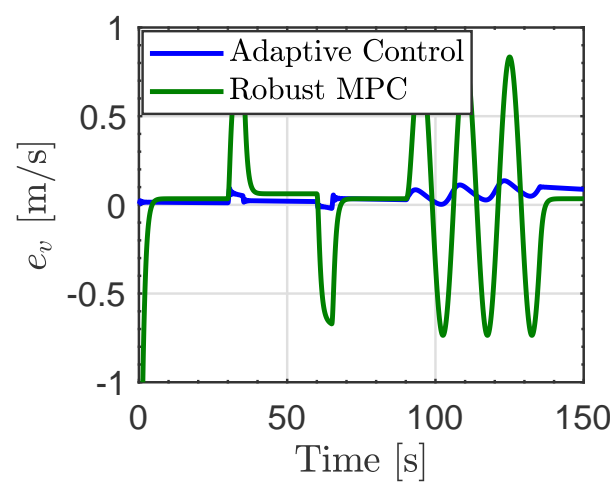

(a)

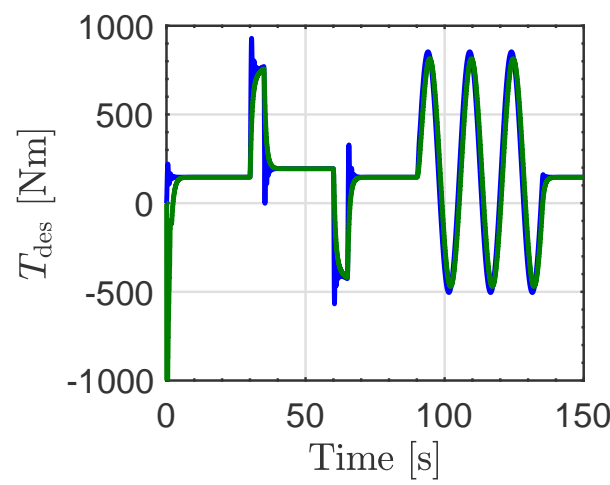

(c)

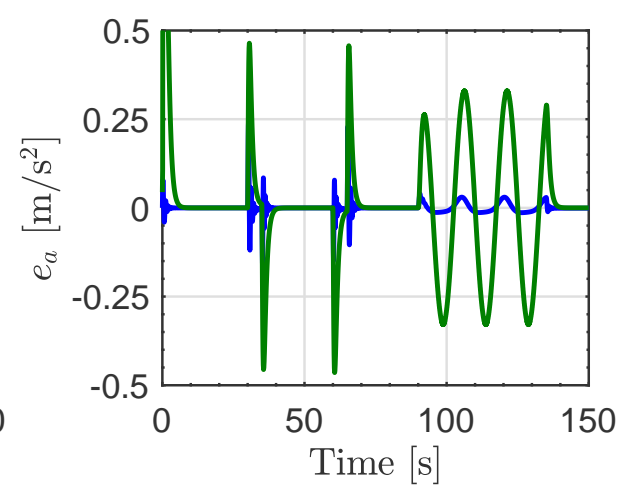

(b)

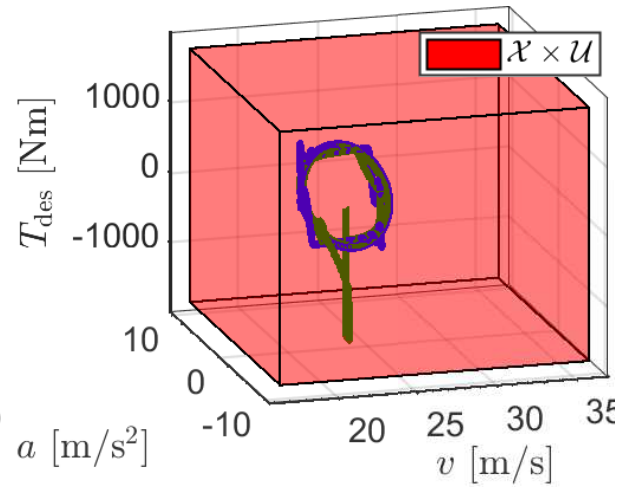

(d)

Fig. 4: RMPC (green) and Adaptive Control (blue line) numerical results. a) velocity error, b) acceleration error, c) control action (driving/braking torque), and d) phase portrait.

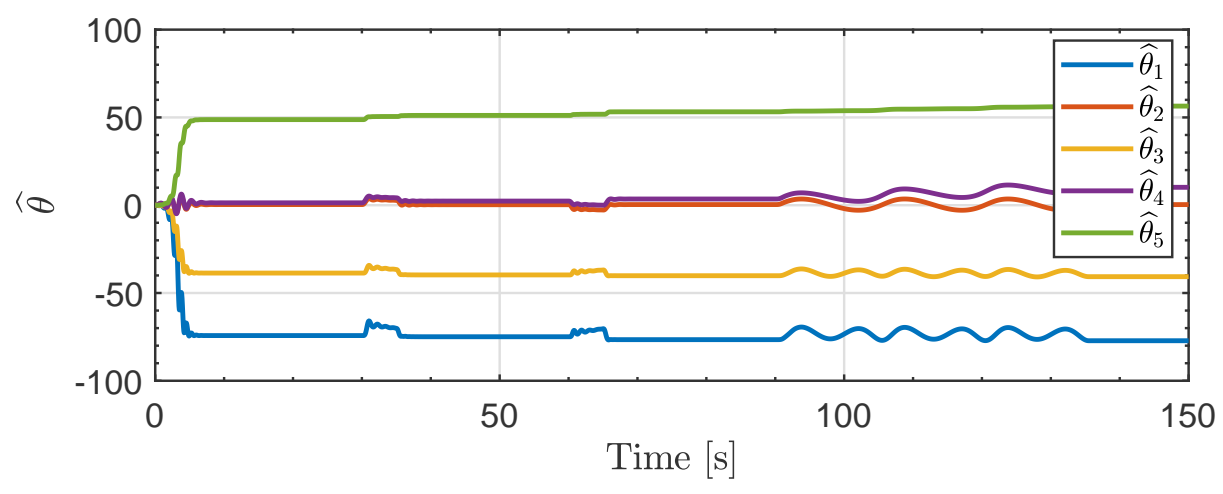

Fig. 5: Adaptive control results. Adaptive gains 


\section{References}

1. U. Montanaro, S. Dixit, S. Fallah, M. Dianati, A. Stevens, D. Oxtoby, and A. Mouzakitis, "Towards connected autonomous driving: review of use-cases," Vehicle System Dynamics, vol. 57, no. 6, pp. 779-814, 2019.

2. S. E. Li, Y. Zheng, K. Li, and J. Wang, "An overview of vehicular platoon control under the four-component framework," in 2015 IEEE Intelligent Vehicles Symposium (IV). IEEE, 2015, pp. 286-291.

3. F. Gao, S. E. Li, Y. Zheng, and D. Kim, "Robust control of heterogeneous vehicular platoon with uncertain dynamics and communication delay," IET Intelligent Transport Systems, 2016.

4. U. Montanaro, S. Fallah, M. Dianati, D. Oxtoby, T. Mizutani, and A. Mouzakitis, "On a fully self-organizing vehicle platooning supported by cloud computing," in International Conference on Internet of Things: Systems, Management and Security.

5. F. Gao, D. F. Dang, S. Huang, and S. E. Li, "Decoupled robust control of vehicular platoon with identical controller and rigid information flow," International Journal of Automotive Technology, 2017.

6. F. Angulo, M. di Bernardo, U. Montanaro, A. Rincon, and S. Santini, "Adaptive control for state dependent switched systems in Brunovsky form," in 2013 European Control Conference (ECC). IEEE, 2013, pp. 3712-3717.

7. I. Alvarado, D. Limón, T. Alamo, M. Fiacchini, and E. F. Camacho, "Robust tube based MPC for tracking of piece-wise constant references," in 2007 46th IEEE Conference on Decision and Control, 2007, pp. 1820-1825.

8. Y. Zheng, S. E. Li, K. Li, and L.-Y. Wang, "Stability margin improvement of vehicular platoon considering undirected topology and asymmetric control," IEEE Transactions on Control Systems Technology, vol. 24, no. 4, pp. 1253-1265, 2015.

9. J. B. Rawlings and D. Q. Mayne, Model predictive control: Theory and design. Nob Hill, 2009.

10. S. V. Rakovic, E. C. Kerrigan, K. I. Kouramas, and D. Q. Mayne, "Invariant approximations of the minimal robust positively invariant set," IEEE Transactions on Automatic Control, vol. 50, no. 3, pp. 406-410, 2005.

11. S. Dixit, U. Montanaro, M. Dianati, D. Oxtoby, T. Mizutani, A. Mouzakitis, and S. Fallah, "Trajectory Planning for Autonomous High-Speed Overtaking in Structured Environments Using Robust MPC," IEEE Transactions on Intelligent Transportation Systems, pp. 1-14, 2019. 